\title{
A Review on Anticancer Potential of Natural Drugs: Hispolon and Limonene
}

\author{
K. Chandra Sekhar ${ }^{1,2}$, A. Rajanikanth ${ }^{1}$, Md. Nazneen Bobby ${ }^{2}$ and \\ Jagadeeswara Reddy Kanala ${ }^{1}$. \\ ${ }^{1}$ Sugen Life Sciences Pvt.Ltd, Tirupati-517502, AP, India \\ ${ }^{2}$ Department of Biotechnology, Vignan University, Vadlamudi, Guntur-522213, AP, India
}

*Corresponding author

\section{A B S T R A C T}

\section{Keywords}

Cancer cells, Natural products, Hispolon, Limonene, Carcinoma

Article Info

Accepted:

26 October 2018

Available Online:

10 November 2018
Cancer is one of the leading causes of death and globally the numbers of cases of cancer are increasing gradually. There are several medicines available in the market to treat the various types of cancer but no drug is found to be fully effective and safe. The major problem in the cancer chemotherapy is the toxicity of the established drugs. However natural products have proved effective and safe in the treatment and management of cancers. These days most of the research work on cancer drugs is targeted on bio based natural products. Many natural products and their analogues have been identified as potent anti-cancer agents and day by day the anti-cancer property of various bio based compounds is being identified. Here an attempt is being made through this review to highlights the efficacy of Hispolon and Limonene and their analogues established as anticancer agents.

\section{Introduction}

Cancer is a major public health burden in both developed and developing countries. It is an abnormal growth of cells in body that can lead to death and globally the numbers of cancer patients are increasing day by day.

Many natural products and their analogues have been identified as potent anti-cancer agents and day by day the anti-cancer property of various plants and fungi is being identified. New drug discovery is time consuming \&laborious process. So the anticancer activity of certain natural products and their analogs can be enhanced by synthesizing new derivatives based on active pharmacophore models; drug resistance, solubility and metabolic limitations can be overcome by appropriate molecular modification.

In this context, natural products derived from the biological sources have emerged as the first choice of researchers. Accordingly, several plant-/fungal-/bacterial-derived natural products are in different stages of evaluation as new therapeutic agents (Veeresham, 2012 and Bernardini et al., 2017). There has been also a growing interest among the researchers for exploring synthetic derivatives of natural products as a novel class of drugs with multiple activities and target specificity. In 
this review, we summarize several studies that show the anticancer effects of Hispolon and Limonene.

\section{Hispolon}

Hispolon (HS) is a bioactive polyphenol found in several medicinal mushrooms. It was isolated initially from Inonotus hispidus (Ali et al., 1996) subsequently; it was isolated from other species of mushrooms such as Phellinus linteus and Phellinus igniarius (Mo et al., 2004 and Wang et al., 2014). There are increasing lines of evidence in the literature to suggest that Hispolon exhibits a wide range of medicinal properties. For example, it has been reported for antiviral (Ali et al., 2003), hepatoprotective (Huang et al., 2012), immunomodulatory (Chang et al., 2011) and ant proliferative activities (Huang et al., 2011, Chen et al., 2013 and $\mathrm{Wu}$ et al., 2014) in diff erent models. Of these, antitumor activity of Hispolon has been studied in detail by different groups and it was observed that it inhibits growth of cancer cells through induction of cell cycle arrest, apoptosis, and suppression of metastasis (Chen et al., 2013, Chen et al., 2008, Huang et al., 2010 and Hsiao et al., 2013).However, the underlying mechanisms responsible for these eff ects are not understood completely. Considering the importance of Hispolon as a pharmacological agent, several of its new derivatives have been synthesized and evaluated for antiproliferative and antitubercular eff ects.

On similar lines, recently Pogakula Chethna et al., reported the abilities of HS and its derivatives such as hispolonpyrazole (HP), hispolonmonomethyl ether (HME), and hispolonmonomethyl ether pyrazole (HMEP) to scavenge reactive oxygen species (ROS) and to exhibit antioxidantactivity in cell-free systems. The exposure of carcinogenic agents such as radiation, pollutants, and xenobiotics are known to increase the intracellular levels of ROS, leading to oxidative damages. Although DNA is a stable, well-protected molecule, it is the most critical subcellular target of ROS-induced oxidative damages and is believed to be responsible for the onset of cell death, mutagenesis, and carcinogenesis. Therefore, any agent which can attenuate ROS (antioxidant activity) and the subsequent DNA damage (antigenotoxic activity) can help in reducing the initiation and progression of cancer. The structural formula of hispolon is shown in Figure 1.

\section{Lung cancer}

Q. Wu et al., (2014) examined the effects of hispolon on the lung cancer A549 and H661 cells. According to them Hispolon decreased cell viability in a dose- and time-dependent manner. The cell cycle distribution showed that hispolon enhanced the accumulations of the cells in G0/G1 phase. Mechanically, hispolon decreased the expression of G1-S transition-related proteins: Cyclin D1, cyclin E, CDK2, CDK4 and CDK6, but increased the expression of CDK inhibitor p21CIP1 and p27KIP1. Moreover, hispolon induced cell apoptosis through activation of the mitochondrial pathway, evidenced by the loss of mitochondrial membrane potential, the release of cytochrome c into cytosol, and the cleavage of caspase-9, caspase-3 and poly (ADP-ribose) polymerase (PARP) in hispolontreated cells. Additionally, hispolon enhanced the expression of $\mathrm{p} 53$, specific silencing of which almost completely reversed hispolonmediated antitumor activity.

Moreover, hispolon treatment was more effective on H661 cells than on A549 cells in inhibiting cell viability and inducing cell apoptosis. They suggested that hispolon inhibits the cell viability, induces G0/G1 cell cycle arrest and apoptosis in lung cancer cells and p53 plays a critical role in hispolonmediated antitumor activity. 


\section{Breast and bladder cancer}

MDM2 proto oncogene is overexpressed in many human tumors, including breast and bladder cancers. Therefore, downregulation of MDM2 has been considered an attractive therapeutic strategy. Hispolon extracted from Phellinus species was found to induce epidermoid and gastric cancer cell apoptosis. However, the mechanisms are not fully understood. T.-L. Lu et al., (2009) reported that hispolon inhibited breast and bladder cancer cell growth, regardless of p53 status. Furthermore, p21WAF1, a cyclin-dependent kinase inhibitor, was elevated in hispolontreated cells. MDM2, a negative regulator of p21WAF1, was ubiquitinated and degraded after hispolon treatment. They also found that activated ERK1/2 (extracellular signalregulated kinase1/2) was recruited to MDM2 and involved in mediating MDM2 ubiquitination. Based on this finding, we investigated whether the sensitivity of cells to hispolon was related to ERK1/2 activity. The results indicated that cells with higher ERK1/2 activity were more sensitive to hispolon. In addition, hispolon-induced caspase-7 cleavage was inhibited by the ERK1/2 inhibitor, U0126. They concluded that hispolon ubiquitinates and downregulates MDM2 via MDM2recruited activated ERK1/2. Therefore, hispolon may be a potential anti-tumor agent in breast and bladder cancers.

\section{Gastric cancer}

W. Chen et al., (2008) investigated the efficacy of hispolon in human gastric cancer cells and explored the cell death mechanism. Hispolon induced ROS-mediated apoptosis in gastric cancer cells and was more toxic toward gastric cancer cells than toward normal gastric cells, suggesting greater susceptibility of the malignant cells. The mechanism of hispolon induced apoptosis was that hispolon abrogated the glutathione antioxidant system and caused massive ROS accumulation in gastric cancer cells. Excessive ROS caused oxidative damage to the mitochondrial membranes and impaired the membrane integrity, leading to cytochrome $\mathrm{c}$ release, caspase activation, and apoptosis. Furthermore, hispolon potentiated the cytotoxicity of chemotherapeutic agents used in the clinical management of gastric cancer.

\section{Oral cancer}

Hispolon from Phellinus linteus is being increasingly used to treat a wide variety of disease processes such as oral ulcer, gastroenteric disorder, inflammation, lymphatic disease, and various cancers. However, the mechanism underlying its antioral cancer effect is poorly understood. W. Chen et al., (2006) prepared the ethanol extract of Phellinus linteus as a crude drug, and then obtained the active component hispolon by bioassay-guided isolation. Hispolon showed a dose dependent inhibition of human epidermoid KB cell proliferation with IC50 of $4.62 \pm 0.16 \mathrm{~g} / \mathrm{ml}$. Furthermore, it was revealed that hispolon could induce human epidermoid KB cell apoptosis with the characteristic of a DNA ladder, and with a significant increase of sub-G1. This process was accompanied by the collapse of mitochondrial membrane potential, the release of cytochrome $c$ and the activation of Caspase-3. Results revealed that hispolon induced the death of $\mathrm{KB}$ cells through a mitochondria mediated apoptotic pathway.

\section{Nasal cancer}

Nasopharyngeal carcinoma (NPC) is the most common cancer originating in the nasopharynx, where the nasal passages and auditory tubes join the remainder of the upper respiratory tract. NPC occurs in children and adults. It is a distinct entity of head and neck cancers because of its characteristic 
epidemiology, pathogenesis, and association with the Epstein-Barr virus. Importantly, it is ametastasis of cancer cells to the neck lymph nodes, which can occur in up to $75 \%$ of NPC patients, which represents an adverse prognostic factor of the disease. Hispolon, a phenol compound isolated from Phellinus linteus (PL), possesses anti-inflammatory, anti-proliferative, and antioxidant effects. However, the effects of hispolon on human nasopharyngeal carcinomas have yet to be evaluated. The molecular mechanism by which hispolon anticancer effects in human nasopharyngeal carcinomas cells were investigated by Hsieh et al., (2014). The results showed thathispolon significantly inhibited cell proliferation of HONE-1 and NP-039 cell lines. Furthermore, hispolon induced apoptosis throughcaspases- $3,-8$, and 9 activations and PARP cleavage in dose- and time-dependent manner in HONE-1 andNP039 cells. Moreover, hispolon also showed that increase phosphorylation of ERK1/2, p38 MAPK andJNK1/2 in dose- and timedependent manner by western blot analysis. However, hispolon-induced activation of the caspase- $-3,-8$ and -9 significantly abolished by inhibition of p38 MAPK and JNK1/2 specific inhibitors. The effects of hispolon on the apoptosis and related regulation mechanism in HONE-1 and NPC-039 cells takes place and hispolon may be a useful candidate as a chemotherapeutic agent for NPC therapy.

\section{Liver cancer}

Hepatocellular carcinoma (HCC) is a lethal cancer, several etiologic factors, including exposure to aflatoxinB1 and infection with hepatitis $B$ and $C$ viruses have been classified as high-risk factors associated with HCC. Guan-Jhong Huang et al., (2011) investigated the anti-proliferative eff ect of hispolon on human hepato cellular carcinoma Hep3B cells by using the MTT assay, DNA fragmentation, DAPI (4,6-diamidino-2- phenylindoledihydrochloride) staining, and flow cytometric analyses. Hispolon inhibited cellular growth of Hep3B cells in a timedependent and dose-dependent manner, through the induction of cell cycle arrest at $S$ phase measured using flow cytometric analysis and apoptotic cell death, as demonstrated by DNA laddering. Hispoloninduced S-phase arrest was associated with a marked decrease in the protein expression of cyclins $\mathrm{A}$ and $\mathrm{E}$ and cyclin-dependent kinase (CDK) 2, with concomitant induction of p21waf1/Cip1 andp27Kip1. Exposure of Hep3B cells to hispolon resulted in apoptosis as evidenced by caspase activation, PARP cleavage, and DNA fragmentation. Hispolon treatment also activated JNK, p38 MAPK, and ERK expression. Inhibitors of ERK (PB98095), but not those of JNK (SP600125) and p38MAPK (SB203580), suppressed hispolon-induced S-phase arrest and apoptosis in Hep3B cells. These findings established a mechanistic link between the MAPK pathway and hispolon-induced cell cycle arrest and apoptosis inHep3B cells.

\section{Colon cancer}

The ability of hispolon to modulate Tumour necrosis factor-related apoptosis-inducing ligand (TRAIL) induced apoptosis in human colon cancer cells was investigated by Kim et al., (2016) as well as the subsequent mechanism of action. Hispolon was found to enhance TRAIL-induced apoptosis through the upregulation of pro-apoptotic proteins, downregulation of cell survival proteins and upregulation of death receptors. Hispolon was found to activate caspase- 3 , caspase- 8 and caspase-9, while downregulating the expression of cell survival proteins such as cFLIP, Bcl-2 and Bcl-xL and upregulating the expression of Bax and truncated Bid. They also found hispolon induced death receptors in a non- cell type- specific manner. Upregulation of death receptors by hispolon 
was found to be p53-independent but linked to the induction of CAAT enhancer binding protein homologous protein (CHOP). Overall, hispolon was demonstrated to potentiate the apoptotic effects of TRAIL through downregulation of anti-apoptotic proteins and upregulation of death receptors linked with CHOP and pERK elevation.

Similarly Balaji et al., (2015) synthesized hispolon and its analogues, their cytotoxicities were evaluated in human prostate, colon and breast cancer cell lines in comparison to noncancer canine kidney, mouse embryonic fibroblast and human primary embryonic kidney cell lines using the MTT assay. Among the 26 hispolonanalogs synthesized and evaluated, compound VA-2 was the most active, showing significant concentrationdependent cytotoxicity and selectivity in the colon followed by prostate cancer cell lines, with the observed mean IC50 values in the range 1.4 to $6.3 \mu \mathrm{M}$. Compared to hispolon, compound VA-2 was 3.7 to 4.7 times and 2 to 5.5 times more potent in the colon and prostate cancer cell lines, respectively. In addition, compound VA-2 induced a significant concentration-dependent reduction in mitochondrial membrane potential resulting in pre-apoptosis and apoptosis in the HCT-116 colon cancer cell line. Hispolonanalogs activity varied significantly when the aromatic functional groups and $\beta$-diketone functional groups are modified. As a result VA-2, hispolonanalogs VA-4, VA-7 and VA-15 also showed prominent activity on these cell lines.

Fig.1 Two (1A) and three (1B) dimensional structure of hispolon

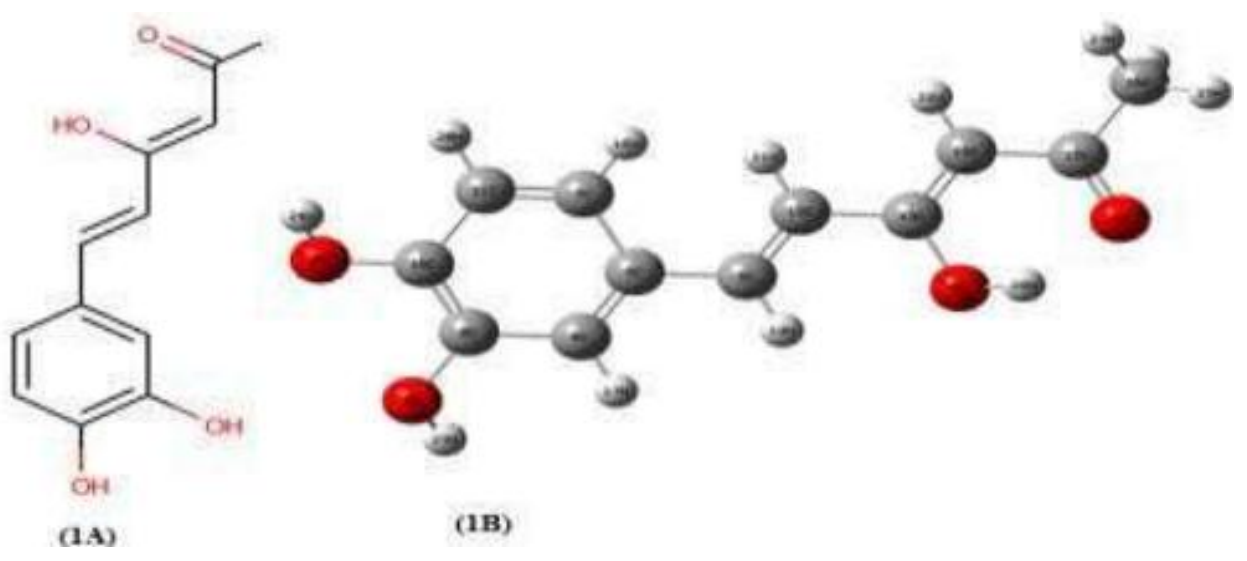

Fig.2 Chemical structure of Limonene

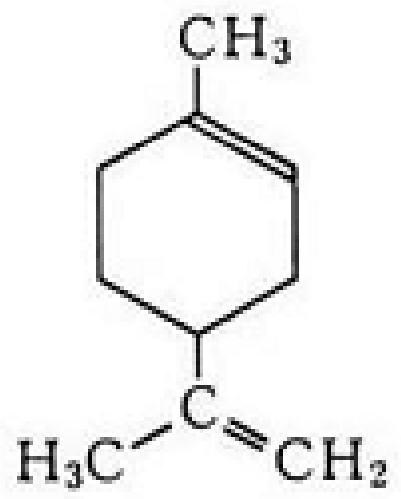




\section{Leukemia cancer}

Y.-C. CHEN et al., (2013) investigated the antiproliferative effect of hispolon on human hepatocellular carcinoma NB4 cells using the MTT assay, DNA fragmentation, DAPI (4, 6diamidino-2-phenylindole dihydrochloride) staining, and flow cytometric analysis. Hispolon inhibited the cellular growth of NB4 cells in a dose-dependent manner through the induction of cell cycle arrest at G0/G1 phase measured using flow cytometric analysis and apoptotic cell death, as demonstrated by DNA laddering. Exposure of NB4 cells to hispoloninduced apoptosis-related protein expressions, such as the cleavage form of caspase 3, caspase 8, caspase 9, poly (ADP ribose) polymerase, and the proapoptoticBax protein. Western blot analysis showed that the protein levels of extrinsic apoptotic proteins (Fas and FasL), intrinsic related proteins (cytochrome c), and the ratio of Bax/Bcl-2 were increased in NB4 cells after hispolon treatment. Hispolon-induced G0/G1-phase arrest was associated with a marked decrease in the protein expression of $\mathrm{p} 53$, cyclins D1, and cyclins E, and cyclin-dependent kinases (CDKs) 2, and 4, with concomitant induction of p21waf1/Cip1 and p27Kip1. Hispolon induces both of extrinsic and intrinsic apoptotic pathways in NB4 human leukemia cells in vitro.

\section{Limonene}

D-limonene (1-methyl-4- (1-methylethenyl) cyclohexane) is a monocyclic monoterpene with a lemon-like odour and is a major constituent in several citrus oils (orange, lemon, mandarin, lime, and grapefruit). It is listed in the Code of Federal Regulation as generally recognized as safe and widely used as flavouring and fragrance additive in food industry (Jidong Sun, 2007). Limonene is colourless liquid with a molecular weight of 136.24 (molecular formula: C10H16: CAS-
No. 5989-27-5) (Fig. 2). Limonene is shown to have wide clinical applications in cancer as well as in other disease conditions. It is used to dissolve cholesterol containing gallstones (Igimi et al., 1976) and due to its potential for neutralizing gastric acid, it has also been used to relieve heartburn and gastroesophageal reflux disorder (Wilkins, 2002). In preclinical cancer models, it has been shown to prevent or delay the growth of a number of cancer types including lymphomas (Del Toro Arreola et al., 2005), mammary (Maltzman et al., 1989), gastric (Lu XG et al., 2004), liver (Kaji et al., 2001), lung (Raphaeland Kuttan, 2003) and prostate cancer (Rabiand Bishayee, 2009).

\section{Colon cancer}

Raja Ratna Reddy et al., (2016) investigated synergistic anticancer effects of limonene and BEZ235 combination in COLO-320 and HCT-116 colon cancer cells. Cells were treated with both the drugs alone and in combination and the effects on cell viability; cell migration and clonogenic potential were examined. Results show that both drugs exhibited dose and time dependant cytotoxicity on the cell lines tested. Composing analysis of the drug combination effects revealed the strong synergistic interaction of the combination. These results also indicate that COLO-320 cells were more sensitive for anticancer effects of the drugs than HCT-116 cells. The presence of Ras and PI3K mutations in HCT-116 cells could possibly be one of the main reasons for the observed outcome as compared to the wild type expressions of them in COLO-320 cells.

JIA et al., (2013) investigated the effects of D-limonene on colon cancer cell viability and its potential mechanism of action in vitro. After $48 \mathrm{~h}$ of treatment, D-limonene suppressed the viability of LS174T cells in a dose-dependent manner and caused a dose- 
dependent apoptotic cell death. D-limonene activated caspase- 3 and -9 and PARP cleavage in a dose-dependent manner. Moreover, an increase in Bax protein and cytosol cytochrome c from mitochondria and a decrease in Bcl-2 protein were observed following treatment with D-limonene. In addition, D-limonene decreased the levels of p-Akt (Ser473), p-Akt (Thr308) and p-GSK$3 \beta$ (Ser9), suggesting that D-limonene induced apoptosis via the mitochondrial death pathway and the suppression of the PI3K/Akt pathway.

\section{Leukemia cancer}

J. Ji et al., (2006) investigated the proliferation and apoptosis induction by $\mathrm{d}$ limonene in human leukemia cell lines. The results revealed that d-limonene induces adose- and time-dependent apoptosis through an increase of Bax protein expression. They also demonstrate activation of the mitochondrial death pathway, characterized by a release of cytochrome $\mathrm{c}$ and activation of caspase-9 and cleaved caspase-3, but notcaspase-8. Furthermore, both broadspectrumcaspase inhibitor and caspase-3specific inhibitor succeed in attenuating $d$ limonene-induced apoptotic cell death. These observations suggest that d-limonene induces apoptosis in both tested human leukemia cells via acaspase-dependent mitochondrial death pathway.

\section{Skin cancer}

Chaudhary et al., (2012) investigated the effects of D-limonene on the growth of 7,12dimethylbenz[a] anthracene (DMBA) initiated and 12-O-tetradecanoylphorbol-13acetate (TPA) -promoted skin tumor development. They found that D-limonene (50 and $100 \mathrm{mg} / \mathrm{kg}$ body weight) treatments to the mouse skin significantly reduced the TPA-induced (a) edema and hyperplasia ( $\mathrm{p}<$
0.001); (b) expression of cyclooxygenase-2; (c) ornithine decarboxylase activity ( $\mathrm{p}<$ $0.001)$; and $(\mathrm{d})[3 \mathrm{H}]$ thymidine incorporation into DNA ( $p<0.001)$. In addition, treatment of D-limonene effectively restored the level of reduced glutathione, glutathione peroxidase, glutathione reductase, glutathione S-transferase, catalase and malondialdehyde production in TPA-treated mouse skin. In a two-stage skin tumorigenesis study, Dlimonene significantly reduced the tumor burden $(\mathrm{p}<0.005)$ and tumor incidence as compared to DMBA/TPA-treated mice. DLimonene treatment also extended the latency period of tumor development from 4 to 9 weeks. D-Limonene treatment decreased the expression level of Ras, Raf and phosphorylation of extracellular signalregulated protein kinase $1 / 2$ in DMBA/TPAinduced tumors. A decrease in the expression of Bcl-2 and an increase in Bax expression were also observed in tumor tissues of mice treated with D-limonene. These findings suggest that D-limonene may exert its chemopreventive activity through the inhibition of inflammation, oxidative stress and Ras-signaling as well as the induction of pro-apoptotic state during TPA-mediated promotion of DMBA-induced skin cancer in mouse model.

\section{Pancreatic cancer}

The effect of d-limonene on pancreatic carcinogenesis induced by $\mathrm{N}$-nitrosobis (2oxopropyl) amine (BOP) was investigated in Syrian golden hamsters by Nakaizumi et al., (1997). During and after 5 weekly injections of BOP, each hamster was fed diet containing d-limonene. In week 26, quantitative histological analysis showed that prolonged treatment with d-limonene significantly reduced the number of pancreatic carcinomas. Administration of d-limonene did not cause a significant increase in the apoptotic index, but caused a significant decrease in the 
BrdUlabeling index of carcinoma. These findings indicate that d-limonene inhibits the development of pancreatic carcinoma not by enhancing tumor cell loss through apoptosis, but rather by inhibiting cell proliferation.

\section{Mammary cancer}

C.E. Elson et al., (1988) reported that the monoterpenoid d-limonene is effective in preventing 7,12-dimethylbenz[a] anthracene $\left(\mathrm{DMBA}^{*}\right)$-induced mammary cancer in rats when incorporated in the diet from 1 week before carcinogen administration through to the end of the follow-up period. A dose as low as 20 p.p.m. was found to be effective while no toxicity was observed at dosesas high as 100000 p.p.m. in the diet. They determine the effectiveness of dietary $d$ limonene when fed only during the initiation or during the promotion/progression stage of DMBA-induced mammary carcinogenesis. They conclude that d-Limonene was found to be effective in reducing the average number of rat mammary carcinomas that developed in 7,12-dimeihylbenz[a] anthracene-treated rats when the terpene was fed during the initiation or during the promotion/progression stage of carcinogenesis. The time to the appearance of the first tumor was extended only when dlimonene was fed during the initiation stage. These effects could not be attributed to changes in mammary-relevant endocrine functions

\section{Prostate cancer}

Clinical trials have shown that docetaxel combined with other novel agents can improve the survival of androgen-independent prostate cancer patients. d-Limonene, a nonnutrient dietary component, has been found to inhibit various cancer cell growths without toxicity. Thangaiyan Rabi and AnupamBishayee (2009) sought to characterize whether a non-toxic dose of d- limonene may enhance tumor response to docetaxel in an in vitro model of metastatic prostate cancer. d-Limonene and docetaxel in combination significantly enhanced the cytotoxicity to DU-145 cells than PZ-HPV-7 cells. Exposure of DU-145 cells to a combined d-limonene and docetaxel resulted in higher ROS generation, depletion of GSH, accompanied by increased caspase activity than docetaxel alone. It also triggered a series of effects involving cytochrome c, cleavages of caspase-9, 3 and poly (ADP-ribose) polymerase, and a shift in Bad: Bcl-xL ratio in favor of apoptosis. Apoptotic effect was significantly blocked on pretreatment with Nacetylcystein, indicating that antitumor effect is initiated by ROS generation, and caspase cascades contribute to the cell death. Results show, for the first time, that d-limonene enhanced the antitumor effect of docetaxel against prostate cancer cells without being toxic to normal prostate epithelial cells. The combined beneficial effect could be through the modulation of proteins involved in mitochondrial pathway of apoptosis. DLimonene could be used as a potent non-toxic agent to improve the treatment outcome of hormone-refractory prostate cancer with docetaxel.

This review paper provides information on natural products with potential to decrease growth of cancer or be used as adjuvant with cancer treatments for patients who already have or have had cancer. It is documented that natural products such as Hispolon and Limonene have rich anticancer potential, and they have exhibited anticancer activity in animal models as well as human cell lines. Information from this review health care professional can initiate discussion with colleagues to determine whether patient may benefit from taking a specific natural product. Selected natural products have been explored for biological activity and further investigations into anticancer activity of the 
biological compounds showing promising activity, must be undertaken.

\section{References}

Akihiko Nakaizumi, Miyako Baba, Hiroyuki Uehara, Hiroyasu Iishi, and Masaharu Tatsuta. 1997. Limonene inhibits Nnitrosobis (2-oxopropyl) amine induced hamster pancreatic carcinogenesis. Cancer Letters. 117: 99-103.

Ali, N. A. A. Jansen, R. Pilgrim, H. Liberra, K. andLindequist, U. 1996. Hispolon, a yellow pigment from Inonotushispidus. Phytochemistry. 41: 927-929.

Ali, N. A. A. Mothana, R. A. A. Lesnau, A. Pilgrim, H. and Lindequist, U.2003.Antiviral activity of Inonotushispidus.Fitoterapia.74: 483-485.

Bernardini, S. Tiezzi, A. LaghezzaMasci, V. and Ovidi, E. 2017. Naturalproducts for human health: an historical overview of the drug discovery approaches. Nat. Prod. Res. 27:1-25.

Chang, H.Y. Sheu, M.J. Yang, C.H. Lu, T.C. Chang, Y.S. Peng, W.H. Huang, S.S. and Huang, G.J. 2011. Analgesic effects and the mechanisms of anti-inflammation of hispolon in mice. J. Evidence-Based Complementary Altern. Med. 478246.

Charles E. Elson, Terese H. Maltzman, Jami L. Boston, Martin A. Tanner and Michael N. Gould. 1988. Anti-carcinogenic activity of d-limonene during the initiation and promotion/progression stages of DMBAinduced rat mammary carcinogenesis. Carcinogenesis. 9 (2): 331-332.

Chaudhary, S.C. Siddiqui, M.S. Athar, M. and SarwarAlam, M. 2012. D-Limonene modulates inflammation, oxidative stress and Ras-ERK pathway to inhibit murine skin tumorigenesis. Human and Experimental Toxicology.1-14.

Chen, W. Zhao, Z. Li, L. Wu, B. Chen, S.F. Zhou, H. Wang, Y. and Li, Y.Q. 2008. Hispolon induces apoptosis in human gastric cancer cells through a ROS- mediated mitochondrial pathway. Free Radical Biol.Med. 45: 60-72.

Chen, Y.C. Chang, H.Y. Deng, J.S. Chen, J.J. Huang, S.S. Lin, I.H. Kuo, W.L. Chao, W. and Huang, G.J. 2013. Hispolon from Phellinus linteus induces G0/G1 cell cycle arrest and apoptosis in NB4human leukaemia cells. Am. J. Chin. Med. 41: 1439-1457.

Del Toro Arreola S, Flores Torales E, Torres Lozano C, Del Toro Arreola A, TostadoPelayo K, Guadalupe Ramirez Duenas $\mathrm{M}$, and Daneri Navarro A. 2005.Effect of D-limonene on immune response in $\mathrm{BALB} / \mathrm{c}$ mice with lymphoma. IntImmunopharmacol. 5: 829838.

Guan-Jhong Huang, Jeng-Shyan Deng, ShyhShyun Huang, and Miao-Lin Hu. 2011. Hispolon Induces Apoptosis and Cell Cycle Arrest of Human Hepatocellular Carcinoma Hep3B Cells by Modulating ERK Phosphorylation. J. Agric. Food Chem. 59: 7104-7113.

Hsiao, P.C. Hsieh, Y.H. Chow, J.M. Yang, S.F. Hsiao, M. Hua, K.T. Lin, C.H. Chen, H.Y. and Chien, M.H. 2013. Hispolon induces apoptosis through JNK1/2mediated activation of a Caspase-8, -9, and-3-dependent pathway in Acute Myeloid Leukemia (AML) cells and inhibits AML Xenograft tumor growth in vivo. J. Agric. Food Chem.61: 10063-10073.

Huang, G.J. Deng, J.S. Chiu, C.S. Liao, J.C. Hsieh, W.T. Sheu, M.J. and Wu, C.H. 2012.Hispolon protects against acute liver damage inthe rat by inhibiting lipid peroxidation, proinflammatory cytokine, oxidative stress and down-regulating the expressions of iNOS, COX-2, and MMP9. J. Evidence-Based Complementary Altern. Med. 480714.

Huang, G.J. Deng, J.S. Huang, S.S. and Hu, M.L. 2011.Hispoloninduces apoptosis and cell cycle arrest of human hepatocellular carcinoma Hep3B cells by modulating ERK phosphorylation. J. Agric.Food Chem. 59: 7104-7113. 
Huang, G.J. Yang, C.M. Chang, Y.S. Amagaya, S. Wang, H.C. Hou, W.C. Huang, S.S. and $\mathrm{Hu}$, M.L. 2010. Hispolon suppresses SK-Hep1 human hepatoma cell metastasis by inhibiting matrix metal-loproteinase2/9 and urokinase-plasminogen activator through thePI3K/Akt and ERK signaling pathways. J. Agric. Food Chem. 58: 9468-9475.

Igimi, H. Hisatsugu, T. and Nishimura, M. 1976. The use of d-limonene preparation as a dissolving agent of gallstones. Am J Dig Dis. 21: 926-939.

Jidong Sun, PhD. 2007. D-Limonene: Safety and Clinical Applications. Alternative Medicine Review.12 (3).

Ji-Hunkim, Yu ChulKim and ByoungduckPark. 2016. Hispolon from Phellinus linteus induces apoptosis and sensitizes human cancer cells to the tumor necrosis factorrelated apoptosis-inducing ligand through upregulation of death receptors. Oncology reports. 35: 1020-1026.

Jun Ji, Li Zhang, Yuan-Yuan Wu, Xiao-Yu Zhu, Su-Qing Lv, and Xi-ZuoSun. 2006. Induction of apoptosis by d-limonene is mediated by a caspase-dependent mitochondrial death pathway in human leukemia cells. Leukemiaand Lymphoma.47 (12): 2617 - 2624.

Kaji, I. Tatsuta, M.Iishi, H. Baba, M. Inoue, A.and Kasugai, H. 2001. Inhibition by Dlimonene of experimental hepatocarcinogenesis in Sprague-Dawley rats does not involve p21 (ras) plasma membrane association. Int $\mathrm{J}$ Cancer. 93: 441-444.

Lu, X.G. Zhan, L.B. Feng, B.A., Qu, M.Y., Yu, L.H. and Xie, J.H. 2004. Inhibition of growth and metastasis of human gastric cancer implanted in nude mice by $\mathrm{D}$ limonene. World J Gastroenterol. 10: 2140-2144.

Maltzman, T.H. Hurt, L.M. Elson, C.E. Tanner, M.A. and Gould, M.N. 1989. The prevention of nitrosomethylurea-induced mammary tumors by D-limonene and orange oil.Carcinogenesis. 10: 781-783.
Ming-JuHsieha, Su-Yu Chiend, YingErhChoucChih-Jung Cheng, Judy Chen, and Mu-Kuan Chen. 2014. Hispolon from Phellinus linteus possesses mediate caspases activation and induces human nasopharyngeal carcinomas cells apoptosis through ERK1/2, JNK1/2 and p38 MAPK pathway. Phytomedicine. 21: 1746-1752.

Mo, S. Wang, S. Zhou, G. Yang, Y. Li, Y. Chen, X. and Shi, J. 2004. Phelligridins C-F: cytotoxic pyrano [4,3-c][2] benzopyran-1,6-dioneand furo[3,2c]pyran-4-one derivatives from the fungus Phellinusigniarius. J. Nat. Prod. 67: 823-828.

Neduri V. Balaji, Modukuri V. Ramani, Arabela G. Viana, Leticia P. Sanglard, Jason White, VanisreeMulabagal, Crystal Lee, Theophilus J. Gana, Nosa O. Egiebor, Gottumukkala V. Subbaraju, and Amit K. Tiwari. 2015. Design, Synthesis and In Vitro Cell-based Evaluation of the Anticancer Activities of Hispolon Analogs. Bioorg Med Chem. 23 (9): 2148-2158.

PogakulaChethna, Shruti S. Iyer, Vishwa V. Gandhi, AmitKunwar, Beena G. Singh, AtanuBarik, Neduri V. Balaji, Modukuri V. Ramani, Gottumukkala V. Subbaraju, and K. Indira Priyadarsini. 2018. Toxicity and Antigenotoxic Eff ect of Hispolon Derivatives: Role of Structure in Modulating Cellular Redox State and Thioredoxin Reductase. ACS omega. 3: 5958-5970.

Qiuge $\mathrm{Wu}$, Yan Kang, Hui Zhang, Hongmin Wang, Yuanhua Liu, Jing Wang. 2014. The anticancer effects of hispolon on lung cancer cells. Biochemical and Biophysical Research Communications. 453: 385-391.

Rabi, T. and Bishayee, A. 2009. D-Limonene sensitizes docetaxel induced cytotoxicity in human prostate cancer cells: generation of reactive oxygen species and induction of apoptosis. J Carcinog.8:9.

Raja Ratna Reddy, Y. Chandra sekhar, P. Bharathnandhan Reddy, K. 
Ramamoorthy, S. Ranga Suresh, S. Yasodha Lakshmi, T. Mani Rajesh, and Damodar Reddy, C. 2016. Limonene and BEZ 235 inhibits growth of COLO-320 and HCT-116 colon cancer cells. International Journal of Drug Delivery. 8: 89-95.

Raphael, T.J. and Kuttan, G. 2003. Effect of naturally occurring monoterpenescarvone, limonene and perillic acid in the inhibition of experimental lung metastasis induced by B16F-10 melanoma cells. J ExpClin Cancer Res. 22: 419-424.

Shu-Sheng Jia, Guang-Peng Xi, Ming Zhang, Yan-Bo Chen, Bo Lei, Xin-ShuDong and Yan-Mei Yang. 2013. Induction of apoptosis by D-limonene is mediated by inactivation of Akt in LS174T human colon cancer cells. Oncology reports. 29: 349-354.

Te-Ling Lu, Guan-Jhong Huang, Te-Jung Lu, Jin-Bin Wu, Chieh-Hsi Wu, Tung-Chuan Yang, Akira Iizuka and Yuh-Fung Chen. 2009. Hispolon from Phellinus linteus has antiproliferative effects via MDM2recruited ERK1/2 activity in breast and bladder cancer cells. Food and Chemical Toxicology. 47: 2013-2021.

ThangaiyanRabi, and AnupamBishayee. 2009. d-Limonene sensitizes docetaxel-induced cytotoxicity in human prostate cancer cells: Generation of reactive oxygen species and induction of apoptosis. Journal of Carcinogenesis. 8: 9.

Veeresham, C. 2012. Natural products derived from plants as a source of drugs. J. Adv. Pharm. Technol. Res. 3: 200-201.
Wang, J. Hu, F. Luo, Y. Luo, H. Huang, N. Cheng, F. Deng, Z. Deng, W. and Zou, K. 2014. Estrogenic and anti-estrogenic activities of hispolon from Phellinuslonicerinus (Bond.). Fitoterapia. 95: 93-101.

Wei Chen, Fei-Yu He, and Yong-Quan Li. 2006. The apoptosis effect of hispolon from Phellinus linteus (Berkeley \& Curtis) Teng on human epidermoid KB cells. Journal of Ethnopharmacology. 105: 280-285.

Wei Chen, Zhao Zhao, Ling Li, Bin Wu, Shi-fei Chen, Hong Zhou, Yong Wang, and Yong-Quan Li. 2008. Hispolon induces apoptosis in human gastric cancer cells through a ROS-mediated mitochondrial pathway. Free Radical Biology \& Medicine. 45: 60-72.

Wilkins, J. Jr.2002. Method for treating gastrointestinal disorder. U.S. Patent (642045).

Wu, Q. Kang, Y. Zhang, H. Wang, H. Liu, and Y. Wang, J. 2014.Theanticancer effects of hispolon on lung cancer cells. Biochem. Biophys. Res. Commun. 453: 385-391.

Yi-Chuan Chen, Heng-Yuan Chang, JengShyan Deng, Jian-Jung Chen, ShyhShyun Huang, I-Hsin Lin, Wan-Lin Kuo, Wei Chao and Guan-Jhong Huang. 2013. Hispolon from Phellinus linteus Induces G0/G1 Cell Cycle Arrest and Apoptosis in NB4 Human Leukaemia Cells. The American Journal of Chinese Medicine. 41 (6): 1439-1457.

\section{How to cite this article:}

Chandra Sekhar, K.A. Rajanikanth, Md. Nazneen Bobby and Jagadeeswara Reddy Kanala. 2018. A Review on Anticancer Potential of Natural Drugs: Hispolon and Limonene. Int.J.Curr.Microbiol.App.Sci. 7(11): 3253-3263. doi: https://doi.org/10.20546/ijcmas.2018.711.375 